\title{
Multilokalität in ländlichen Räumen: (K)Ein neues Phänomen?
}

\author{
Lena Greinke, Linda Lange und Karl Martin Born
}

\subsection{Multilokalität und ländliche Räume in Forschung und Praxis}

Ein steigendes Tempo in der Mobilität und zunehmende kulturelle Vielfalt sorgen für Transformationsprozesse in modernen Gesellschaften, die sich durch den sozialen Wandel weiter ausdifferenzieren (Weiske/Petzold/Zierold 2009: 67). Räumliche Mobilität wird in diesem Zusammenhang zu einem Schlüsselthema der kommunalen Entwicklung (Hesse/Scheiner 2007: 138). Während Multilokalität in der Vergangenheit nur von bestimmten Bevölkerungsgruppen, zum Beispiel in Form der Sommerresidenzen gehobener Gesellschaften, oder in Krisenzeiten gelebt wurde (Schier 2009: 57), führen heute immer mehr Menschen ein Leben an mehreren Orten, sodass es zur gelebten Alltagspraxis wird (DittrichWesbuer/Föbker 2013: 391; Dittrich-Wesbuer/Plöger 2013: 200; Nadler 2014: 393; ARL 2016: 1). Als Folge sich verändernder politischer und wirtschaftlicher Rahmenbedingungen werden nicht nur Lebensstile immer individueller und pluralisieren sich, sondern auch Arbeitswelten aufgrund der Globalisierungs- und Singularisierungsprozesse sowie des Strukturwandels immer flexibler (Hesse/ Scheiner 2007: 139). Zunehmende globale räumliche Arbeitsteilung (ebd.: 138)

\author{
L. Greinke $(\bowtie) \cdot$ L. Lange \\ Institut für Umweltplanung, Leibniz Universität Hannover, Hannover, Deutschland \\ E-Mail: greinke@umwelt.uni-hannover.de \\ L. Lange \\ E-Mail: lange@umwelt.uni-hannover.de \\ K. M. Born \\ Universität Vechta, Vechta, Deutschland \\ E-Mail: karl-martin.born@uni-vechta.de
}


und die Differenzierung der Arbeitsmärkte und Gesellschaften bedingen multilokale Lebensweisen zusätzlich (Reuschke 2009: 31). Vor dem Hintergrund gesteigerter Mobilitätsanforderungen und -möglichkeiten und einer vielfach von Arbeitgeber*innen geforderten Flexibilität von Arbeitnehmer*innen kann dies zu einer Zunahme der multilokalen Lebensweisen führen (Hesse/Scheiner 2007; Hilti 2009; Dittrich-Wesbuer/Plöger 2013). Zudem lösen sich Individuen durch die Individualisierung von Lebensstilen aus traditionellen Vergemeinschaftungsprozessen heraus und Selbstverwirklichungsmöglichkeiten nehmen zu (Beck 1986; Beck/Beck-Gernsheim 1994 in Litter/Stawarz 2013: 403). Darüber hinaus führen neue Informations- und Kommunikations- sowie Transporttechnologien zu Veränderungen von Lebensweisen (vgl. Kramer 2020). Daneben stellen ein zunehmender (internationaler) Massentourismus und die weibliche Emanzipation weitere Gründe dar, die mehrörtige Lebensweisen herbeiführen oder befördern können (Hilti 2013: 17). Die skizzierten Entwicklungen können ihrerseits räumliche Implikationen auf verschiedenen Ebenen bewirken (ARL 2016: 1, Weichhart 2020).

Ziel dieses Beitrags ist es, Multilokalität als Forschungsfeld zu skizzieren. Dazu werden zunächst multilokale Lebensweisen und ländliche Räume als eigenständige Forschungsfelder vorgestellt. Danach wird die Bedeutung mehrörtiger Lebensweisen für ländliche Räume anhand beispielhafter multilokaler Lebenspraktiken aus dem Landkreis Diepholz thematisiert.

\subsubsection{Multilokalität als Forschungsfeld}

Zwar löst „das Zeitalter der Mobilität [...] das Zeitalter der Sesshaftigkeit ab“ (Rolshoven 2007: 157), dennoch ist Mobilität nach wie vor in gewisser Weise mit Immobilität verbunden, Entankerung mit Verankerung und Stillstand mit Bewegung (Sheller/Urry 2006: 3). Die genannten Gegensatzpaare geben Begrifflichkeiten bzw. Zustände wieder, die im Rahmen mehrörtiger Lebensweisen von besonderer Bedeutung sein können: So beispielsweise wenn ein Leben an mehreren Orten als Möglichkeit genutzt wird, um einen vollständigen Umzug (an den Arbeitsort) abzuwenden und damit zugleich eine Form der Verankerung oder Immobilität darstellt. Abgesehen von den skizzierten globalen und gesamtgesellschaftlichen Veränderungen, sind multilokale Lebensführungen ebenso von biografischen Verläufen geprägt. Multilokalität, verstanden als eine Perpetuierung eines Übergangs von einem Ort zum anderen, lässt insbesondere zwei Lebensphasen in den Fokus geraten: Eintritt in Ausbildung und Erwerbstätigkeit außerhalb des Geburtsortes und ebenso das Beenden der Erwerbstätigkeit und die 
damit verbundene Rück- oder Hinwendung an einen anderen Ort. Es lässt sich demnach von „Noch-Nicht-Ganz-Weggewanderten“ und „Noch-Nicht-GanzHin- bzw. Zurückgewanderten" Menschen sprechen, womit sich gleichzeitig auch soziale Mobilitäten verbinden lassen. Multilokale Lebensweisen wurden bereits in einer Vielzahl von Disziplinen untersucht, wobei unterschiedliche methodische und theoretische Bezüge im Fokus standen. Die Anfänge lassen sich in der Mobilitätsforschung und Transnationalitätsforschung finden (ARL 2016: 8). Neben der Familiensoziologie, den Naturwissenschaften und der Medizin wurden auch in der Anthropologie bereits mehrörtige Lebensweisen analysiert (Hilti 2009: 79). Ein gesteigertes Forschungsinteresse an Multilokalität ist zudem in der Geografie, Soziologie und Psychologie zu erkennen (Hesse/Scheiner 2007: 139). Die Multilokalitätsforschung lässt sich aufgrund der vielfältigen Anknüpfungspunkte demnach nicht nur einer Disziplin zuordnen, sondern bietet verschiedene Wege der Annäherung. Die dynamische Entwicklung des Forschungsstandes unterstreicht die hohe Relevanz für Gesellschaft und Stadt zusätzlich (DittrichWesbuer/Plöger 2013: 196).

Zunehmende Flexibilisierungstendenzen sorgen für die Auflösung und Veränderung des Normalarbeitsverhältnisses (Litter/Stawarz 2013: 403). Dieses ist seit den 1960er Jahren vorherrschend männlich dominiert und durch Vollzeitbeschäftigung gekennzeichnet. Spätestens aber seit den 1990er Jahren wird es durch arbeitsrechtliche Veränderungen in Folge befristeter Anstellungen und Leiharbeit abgelöst (Reuschke 2010b: 16). Es entstehen häufigere Arbeitsplatzwechsel, die in der Konsequenz räumliche Mobilität herbeiführen (Litter/Stawarz 2013: 403). In der globalisierten Dienstleistungs- und Wissensgesellschaft beschränken sich diese Flexibilisierungsprozesse nicht länger ausschließlich auf die Phase des beruflichen Einstiegs, sondern etablieren sich zunehmend auch durch Projektarbeiten oder berufsbedingte Delegationen (Herrmann 2005; Reuschke 2010b: 15). Darüber hinaus führen soziale und demografische Veränderungen - rückläufige Geburtenzahlen, die Zunahme von Singlehaushalten und ,eine sinkende Bedeutung des männlichen Familienernährermodells" (Reuschke 2010a: 136) als Folge der Zunahme einer Frauenerwerbstätigkeit - dazu, dass Gesellschaften sich weiter flexibilisieren und die räumliche Mobilität zunimmt.

Multilokale Haushalte sind Haushalte, in denen ein oder mehrere Haushaltsmitglieder mehrörtig leben. Im deutschsprachigen Raum wurden in den 1960er Jahren vor allem Wochenendpendler*innen im Baugewerbe aus regionalökonomischer Perspektive untersucht (Reuschke 2009: 31). Das Wochenendpendeln wurde aber auch in den 1970er Jahren im Bayerischen Wald analysiert (vgl. Breyer 1970). Ebenfalls in den 1970er Jahren wurden besonders berufsbedingte Doppelkarrierepaare in der angloamerikanischen Forschung fokussiert 
(Reuschke 2010b: 35). Insbesondere stand in dieser Zeit auch die Freizeitwohnsitzforschung im Mittelpunkt (vgl. Dienel 2009; Dirksmeier 2012). Im deutschsprachigen Raum wurden seitdem diverse Motivationen multilokaler Lebensweisen analysiert, zum Beispiel multilokal Arbeitende in der Erdöl- und Erdgasindustrie in Russlands Arktis (Saxinger 2020), Flugpersonal (Huchler 2020), multilokale kreative Wissensarbeiter*innen (Nadler 2020), multilokal lebende Studierende (Kramer 2020), Dauercamper*innen (Hilti/Huber 2020) oder berufsbedingt Multilokale in Städten (Leubert 2020). Aktuell hat sich die Multilokalitätsforschung zu einem eigenen Forschungsfeld entwickelt und fokussiert verschiedene Formen und Ausprägungen dieser Lebensweise an mehreren Orten (Nadler/Montanari 2013: 417). Der Schwerpunkt liegt dabei vielfach auf multilokal agierenden Individuen sowie deren Strategien, um das Leben an mehreren Orten auszugestalten (s.a. Dittrich-Wesbuer/Föbker 2013; Dittrich-Wesbuer et al. 2015).

Eine andere Herausforderung stellt bisher die nicht einheitlich verwendete Terminologie in der Multilokalitätsforschung dar. Zwar existieren mehrere Typologien und Einordnungen multilokaler Lebensweisen (z. B. Hesse/ Scheiner 2007; Hilti 2013; Duchêne-Lacroix 2020); es existiert jedoch keine einheitlich verwendete Terminologie oder Typologie zu multilokalen Arrangements und mehrörtigen Lebensmodellen (Reuschke 2010b: 23; ARL 2016: 4). Dies ist darauf zurückzuführen, dass sich Multilokalität als fluide Lebensweise aufgrund schwer zu fassender Temporalität, Distanz und Motivation nur ungenügend in Typologien erfassen lässt, wenngleich Typologien eine komplexitätsreduzierende Funktion haben. Es lassen sich damit einzelne, dem jeweiligen Fokus folgende Nuancen oder Spuren der Realität abbilden. Somit lassen sich für verschiedenen Themenbereiche bereits Typologien finden, zum Beispiel bürgerschaftliches Engagement (Lange 2018), Haushaltstypen (Weiske/Petzold/ Zierold 2009), Lebenswelten multilokal Wohnender (Hilti 2013), Partnerschaftsformen (Peuckert 2012), Haushaltsformen von Shuttles (Reuschke 2010a) oder Typen multilokaler Haushalte (Menzl 2020), die jedoch jeweils nur Teilbereiche abdecken und dadurch schwer vergleichbar oder zu verknüpfen sind.

\subsubsection{Ländliche Räume in der Forschung}

Eher ländlich geprägte Landkreise und Gemeinden innerhalb Deutschlands stehen vor vielfältigen Herausforderungen hinsichtlich der demografischen, wirtschaftsstrukturellen, sozialen und räumlichen Entwicklungen. Dabei stellt der demografische Wandel insbesondere ländliche Räume vor große 
Herausforderungen; zu nennen sind hier u. a. Bevölkerungsrückgänge, die selektive Abwanderung junger Menschen und die damit verbundene zunehmende Alterung der Bevölkerung, unzureichende Erwerbsmöglichkeiten sowie Defizite in der Infrastruktur- und Finanzausstattung der Kommunen (u. a. BMVBS/ BBSR 2009; Hahne 2009; Küpper 2010). Ländliche Räume sind generell durch unterschiedliche Strukturmerkmale und eine stark differierende Dynamik, Vielfalt und Eigenständigkeit gekennzeichnet und in unterschiedlicher Weise vom demografischen Wandel betroffen. Waren ländliche Räume in der Vergangenheit vor allem geprägt von landwirtschaftlicher Produktion (Plieninger et al. 2006: 23), übernehmen sie heute ganz vielfältige Funktionen. Neben der Produktions- und Versorgungsfunktion erfüllen sie auch Wirtschafts- (z. B. Schaffung von Arbeit und Einkommen), Bildungs- und Kultur-, Siedlungs- und Wohn-, Sozialleistungs-, Freizeit- und Erholungs-, Entsorgungs- sowie ökologische Funktionen (Weber 2010: 7). Schon lange gibt es nicht mehr DEN ländlichen Raum als homogene Einheit, weshalb häufig im Plural über diese Räume gesprochen wird (Altrock et al. 2005: 7; Leber/Kunzmann 2006: 60; Milbert 2016: 106). Auch im Kontext der räumlichen Planungen sind ländliche Räume deutlich komplexer als häufig angenommen wird (Altrock et al. 2005: 7). Eine einheitliche Definition existiert weder in der Praxis noch in der Wissenschaft (Baum/Weingarten 2004: 149; Franzen et al. 2008: 1; Born 2011: 4 f.), was auf die Verschiedenheit ländlicher Räume zurückzuführen ist. Es bestehen verschiedene Typologien und Klassifizierungen (z. B. „Raumtypen“ nach BBSR 2010; „Entwicklungstypen ländlicher Räume“ nach Blotevogel 2005; „Landatlas“ nach BMEL 2019), die insbesondere anhand der Bevölkerungsdichte und Lage im Raum die ländlichen Räume beispielsweise in strukturstark/strukturschwach oder peripher/zentrennah gruppieren; durch die Geschichte, demografische Entwicklung, geografische Lage, Wirtschaftsstrukturen sowie Mentalitäten und Traditionen wird die Vielfalt jedoch besonders deutlich (Weber 2010: 6).

Die gängigen Ansätze zur Identifikation und späteren Differenzierung ländlicher Räume sind von zwei Schwierigkeiten geprägt: Zum einen geschieht eine Identifikation ländlicher Räume häufig aus einer Abgrenzung zu urbanen Räumen, was nicht zwangsläufig zu einer Stigmatisierung oder Restkategorisierung führt, aber eher davon geleitet ist, welche Eigenschaften ländliche Räume nicht haben. Es handelt sich mithin also um negative Definitionen. Gleichzeitig vermögen die Abgrenzungs- und Differenzierungsansätze der jüngeren Zeit (z. B. BBSR, Thünen-Institut) nur die kartografische Abbildung bestimmter Indikatoren. Somit wird einem wesentlichen Forschungs- und Interpretationsstrang geografischer Raumforschung nicht genüge getan, da Aspekte der Selbst- und Fremdwahrnehmung bzw. der „Konstruktion“ länd- 
licher Räume nicht berücksichtigt werden. Die sogenannte „Neue Kulturgeographie" bietet als weiteres Interpretationsraster ländlicher Räume kultur- und sozialtheoretische Fragestellungen an, die einerseits streng dichotome zugunsten übergangsbezogener Perspektiven vernachlässigen und andererseits Fragestellungen eingebracht hat, die zunächst in urbanen Kontexten verortet waren (z.B. Obdachlosigkeit, Gentrifizierung etc.). Für unsere Fragestellungen sind die Ansätze der „Neuen Kulturgeographie“ insofern relevant, als dass sie dem Wohnen eine Schlüsselrolle zuweisen (vgl. Dirksmeier 2019: 27). Im Kontext der Beschäftigung mit Multilokalität wäre aber gerade für die Gruppe der „AmenitySeeking"-Multilokalen ${ }^{1}$ deren Konstruktion des Ländlichen zu beachten.

Henkel (2010) weist im Kontext ländlicher Räume auf den sozialen und ökonomischen Wandel hin, der regional unterschiedliche Auswirkungen auf die nachhaltige Entwicklung dieser Räume bewirkt. Folgen des Wandels können sowohl Schrumpfung als auch Wachstum sein: Neben ländlichen Regionen, die durch wirtschaftliche Prosperität, Urbanisierungsdruck und Bevölkerungswachstum gekennzeichnet sind, existieren dünn besiedelte, strukturschwache Regionen, die überwiegend mit den infrastrukturellen Folgen einer negativen Bevölkerungsbilanz - verbunden mit Alterung und Abwanderung junger Menschen auf der Suche nach qualifizierter Arbeit bzw. Ausbildung - konfrontiert sind (Leber/ Kunzmann 2006: 60; Milbert 2016: 105). Diese teilräumlichen Entwicklungen bewirken, dass die bereits vorhandene regionale Spaltung mit einem direkten Nebeneinander von schrumpfenden und wachsenden Gemeinden und Städten in Zukunft weiter voranschreiten wird (Spiegel 2007: 22; Pahl-Weber/Roskamm 2007: 86; Henkel 2016: 94) und verdeutlichen die Diversität ländlicher Räume.

Die Forschung zu ländlichen Räumen konzentriert sich aktuell auf Transformationsprozesse, die insbesondere durch den wirtschaftlichen Strukturwandel und aktuelle sowie zukünftige demografische Entwicklungen ausgelöst werden. In diesem Zusammenhang stellt sich vielerorts ganz konkret die Frage, wie die Versorgung mit Leistungen der technischen und sozialen Infrastrukturen in den Dörfern zukünftig gesichert werden kann. Nahversorgung, Mobilität, Bildung, Gesundheitsversorgung und Pflege sind die Hauptthemenfelder, die in der Forschung zu ländlichen Räumen diskutiert werden (u. a. BMVI 2015; Fachinger/Künemund 2015; Neu 2009). Durch die anhaltend gesteigerten

\footnotetext{
${ }^{1}$ Das bezeichnet sog. „Wohlstandsmultilokale“, also Personen, die sich einen mehrörtigen Lebensstil finanziell leisten können und ihn freiwillig führen.
} 
Mobilitätsmöglichkeiten- und bedürfnisse sowie der zunehmend auf dem Arbeitsmarkt geforderten Flexibilität werden auch in ländlichen Räumen multilokale Lebensstile befördert. Ländliche Räume können dabei den Ausgangspunkt für ein Leben an mehreren Orten darstellen oder auch Destination für Multilokale sein und somit die Räume mit sehr unterschiedlichen Herausforderungen konfrontieren (Greinke et al. 2018; Lange 2018).

\subsection{Multilokalität als Trend (auch) in ländlichen Räumen}

Das Phänomen Multilokalität beschränkt sich nicht nur auf urbane Räume, sondern ist auch in kleinstädtischen Bereichen und ländlichen Räumen zu finden (Dittrich-Wesbuer 2015: 16) und stellt auch Dörfer vor große Herausforderungen. Dabei gewinnen multilokale Lebensweisen durch die allgemein erhöhten Mobilitätsanforderungen und -möglichkeiten an Bedeutung für die räumliche Entwicklung (Dittrich-Wesbuer/Föbker 2013: 391; Dittrich-Wesbuer/Plöger 2013: 197; Dittrich-Wesbuer et al. 2014: 358). Die sozialen, ökonomischen und kulturellen Auswirkungen für die Räume sind aber z. T. noch nicht absehbar (ARL 2016: 19). Während Pendler*innenströme seit jeher als Indikatoren für die strategische Ausrichtung von Kommunen gesehen werden, gewinnt die Auseinandersetzung mit multilokalen Lebensweisen v. a. in Zeiten des demografischen Wandels zunehmend an Bedeutung (u. a. Hesse/Scheiner 2007).

\section{Wohnverhältnisse multilokal lebender Personen}

Ebenso wie die Ursachen und Motive sind auch die Unterkünfte der Multilokalen sehr divers (Dittrich-Wesbuer/Hilti 2020). So gibt es Menschen, die eine Zweitwohnung zum Beispiel am Meer, in den Bergen oder am Arbeitsort haben. Diese Zweitwohnung kann ganz unterschiedlich aussehen. Sie kann sowohl angemietet als auch im eigenen Besitz sein. Zudem gibt es verschiedene Unterkunftsarten, wie einzelne Zimmer in einer Wohngemeinschaft, Einzimmerwohnungen, Ferienhäuser in Ferienhaussiedlungen, Campingwagen auf Dauercampingplätzen etc. (ARL 2016: 4). Des Weiteren zählen Schrebergärten oder mobile Wohncontainer (mobile homes) zu Unterkünften von Multilokalen. Besonders freizeitbedingt werden diese als Übernachtungsmöglichkeit genutzt. Weiterhin leben viele Multilokale in Hotels, Gasthäusern oder Pensionen, die sie regelmäßig und wiederkehrend aufsuchen, wenn sie auf Dienstreisen an denselben Ort sind.

Alle Unterkünfte haben - in Abhängigkeit der individuellen Bedürfnisse diverse Größen und Ausstattungen (vgl. Hilti 2020). Sie reichen von einem Sofa 
in der Wohnung von Freunden oder Bekannten über sehr spärlich ausgestattete Zimmer bis hin zu individuell eingerichteten Miet- und Eigentumswohnungen. Besonders für die Wohnungswirtschaft sind Multilokale deshalb oft schwer zu fassen, weil sie sehr individuelle Ansprüche und Bedürfnisse an ihren Wohnraum stellen (ARL 2016: 9 f.). Allgemeingültige Aussagen zu den Ausprägungen mehrörtiger Lebensweisen können nicht getroffen werden.

Zudem sind die Formen mehrörtiger Lebensweisen sehr divers. Beispielsweise gibt es partnerschaftliche Formen wie die Living Apart Together (LAT), die regelmäßig in der Unterkunft der Partnerin oder des Partners übernachten. Diese Personen haben zumeist zwei getrennte Haushalte zwischen denen sie regelmäßig wechseln (ARL 2016: 4). Darüber hinaus können auch Soldatinnen und Soldaten zur Gruppe der Multilokalen zählen. Sie übernachten in Zimmern in Kasernen und teilen sich diese oft mit anderen Kameradinnen und Kameraden. Überdies führen Seeleute eine mehrörtige Lebensweise und übernachten in der Zeit auf See zumeist in einer Kajüte. Diese teilen sie sich auch häufig mit anderen Seeleuten auf sehr engem Raum auf dem Schiff. Wanderarbeiter*innen oder Saisonarbeitskräfte nutzen beispielsweise häufig Unterkünfte, die sie von ihren Arbeitgebenden zur Verfügung gestellt bekommen. Das sind oft Zimmer in größeren Häusern, die sie sich mit anderen Arbeitskräften teilen. Zum Teil sind es auch Quartiere, die sie bewohnen. Überdies leben beispielsweise Beschäftigte im Bauwesen des Öfteren in Wohncontainern in der Nähe ihrer phasenweisen Arbeitsstelle bzw. auf der Baustelle. Auch kann ein Sofa bei Freunden als Unterkunft dienen, wodurch auch viele Studierenden, die regelmäßig bei Bekannten übernachten, zu den multilokal Lebenden gehören. Zudem übernachten z. B. Großeltern regelmäßig in Gästezimmern der Kinder, um ihre Enkelkinder zu betreuen (vgl. ARL 2016: 4).

\section{Chancen und Herausforderungen von Multilokalität in ländlichen Räumen}

Die Auswirkungen multilokaler Lebensweisen in ländlichen Räumen können in Abhängigkeit der jeweiligen raumstrukturellen Gegebenheiten sehr unterschiedliche Chancen und Herausforderungen herbeiführen. Der demografische Wandel sorgt vielerorts für geringe Geburtenraten, Abwanderungen - v. a. von jungen Menschen - und Überalterung. Daraus resultiert häufig auch ein Rückgang der Versorgungsinfrastrukturen (Hahne 2009: 1; Henkel 2009: 4 ff.; Milbert 2016: 105). Die Folgen dieser Negativspirale können zudem geschwächte Ökonomien (z. B. durch den (Fach-)Arbeitskräftemangel), Gebäudeleerstand, verlassene Ortskerne und ausgedünnte Engagementstrukturen sein (Henkel 2009: 4 ff.; Oel 2009: 129). In diesem Zusammenhang können mehrörtige Lebensweisen sowohl Potenzial als auch Risiko z. B. für den Wohnungsmarkt in ländlichen Räumen sein. Zum Beispiel können Incomings ländliche Räume aufgrund ihrer 
Attraktivität als Destination aufsuchen und damit Leerstand und Entsiedlung durch ihren phasenweisen Zuzug und Zwischennutzungen verringern (Greinke et al. 2018). Oftmals bedeutet Multilokalität aber auch, dass mehrere Wohnungen zunächst in aller Regel die Inanspruchnahme von mehr Wohnfläche ausmachen (Danielzyk/Dittrich-Wesbuer 2020). Vielfach wird zum Beispiel die Familienwohnung auch dann unverändert aufrechterhalten, wenn einer der Verdiener*innen beruflich bedingt eine zusätzliche Wohnung an einem anderen Wohnstandort unterhält.

Zusätzlich zu den Herausforderungen des demografischen Wandels, stehen ländliche Räume häufig vor dem Problem des (Fach-)Arbeitskräftemangels. Besonders junge, gut ausgebildete Personen fehlen zumeist (Franzen et al. 2008: 34). Verstärkt wird diese Herausforderung auf der einen Seite durch Delegationen der Unternehmen in ländlichen Räumen: Oft werden Beschäftigte phasenweise an andere Standorte delegiert und fehlen dann vor Ort. Auf der anderen Seite können solche Delegationen auch dafür sorgen, dass Incomings als (Fach-)Arbeitskräfte zumindest zeitweise in ländliche Räume kommen (Greinke/Hilti 2019).

Das spezifische Nutzungsverhalten von Menschen mit multilokaler Lebensweise hinsichtlich der Inanspruchnahme von Versorgungs- und Daseinsvorsorgeinfrastrukturen ist noch kaum erforscht. Es ist jedoch zu erwarten, dass Menschen ihre Bedarfe zum Beispiel in Bezug auf die Versorgung mit Lebensmitteln innerhalb der Woche an den Orten der temporären Anwesenheit befriedigen und dort zu einer gesteigerten Nachfrage beitragen - während gleichzeitig an Orten, die durch die temporäre Abwesenheit vieler Menschen gekennzeichnet sind, Rückgänge der Umsätze zum Beispiel im Lebensmitteleinzelhandel zu verzeichnen sein dürften (vgl. Weichhart 2020; Petzold 2020). Forschungsansätze, die sich beispielsweise mit der Inanspruchnahme von medizinischen und gesundheitlichen Versorgungsleistungen an den einzelnen Wohnorten der Menschen mit multilokaler Lebensweise beschäftigen, verweisen darauf, dass derartige Leistungen eher am Hauptwohnort und nur in akuten Fällen am Ort der temporären Anwesenheit in Anspruch genommen werden (Leubert 2015). Aufgrund der Altersstruktur der Menschen mit multilokalen Lebensweisen - Studierende, Auszubildende, Menschen im erwerbsfähigen oder Rentenalter - sind Effekte auf besonders kostenintensive (z. B. Kindertagesbetreuungseinrichtungen) bzw. kommunalpolitisch besonders sensible Bereiche der sozialen Infrastrukturen (z. B. Schulen) kaum zu erwarten (vgl. Beitrag Greinke/Albrecht/Othengrafen/ Gutsche/Lehmann in diesem Band (Kap. 5)). Dass Menschen mit Wohnungen in mehreren Orten auch öffentlich finanzierte - und oftmals nicht kostendeckend zu erbringende - Leistungen auch am Nebenwohnort nutzen (z. B. Hallenbäder, Sporteinrichtungen), scheint hingegen unbestritten (ebd). 
Grundsätzlich ist davon auszugehen, dass multilokal lebende Personen und Haushalte zunächst „mehr“ Infrastrukturen nutzen (vgl. auch Weichhart 2020; Scheiner 2020): Dies betrifft Erreichbarkeitsinfrastrukturen, wie Straßen oder ÖPNV-Angebote, die zur Überwindung der Distanzen zwischen den verschiedenen Wohnorten genutzt werden, und geht mit einem erhöhten Energieverbrauch einher (KIT 2015). Besonders in ländlichen Räumen legen Personen oftmals Wege, zum Beispiel zum Einkauf des täglichen Bedarfs oder anderer Güter sowie zur Arbeitsstelle, mit dem PKW zurück (Jacoby/Wappelhorst 2016: 74). Damit können entweder Bedarfe in Bezug auf Straßeninfrastrukturen oder aber hinsichtlich eines bedarfsgerechten ÖPNV-Angebotes - zu dessen Auslastung und Tragfähigkeit die Multilokalen dann grundsätzlich beitragen - verbunden sein (vgl. Beitrag Greinke/Albrecht/Othengrafen/Gutsche/Lehmann in diesem Band (Kap. 5), Danielzyk/Dittrich-Wesbuer 2020).

Neben der Sicherung und Finanzierung von technischer und sozialer Infrastruktur sowie der veränderten Nachfrage auf dem Wohnungsmarkt ist zu erwarten, dass sich multilokale Lebensweisen vor allem auf das bürgerschaftliche Engagement der betroffenen Personen auswirken (Danielzyk/DittrichWesbuer 2020). Unter bürgerschaftlichem Engagement wird die Gesamtheit von freiwilligen, gemeinnützigen und unbezahlten Tätigkeiten bezeichnet (Kahle/ Schäfer 2005), die zugleich das klassische Ehrenamt, d. h. die Übernahme von formell definierten Ämtern und Funktionen im Rahmen von Vereinigungen und Institutionen, umfassen (Becker et al. 2010). Demnach ist „bürgerschaftliches Engagement freiwillig, nicht auf materiellen Gewinn gerichtet, gemeinwohlorientiert, öffentlich beziehungsweise findet im öffentlichen Raum statt, [und] wird in der Regel gemeinschaftlich beziehungsweise kooperativ ausgeübt" (Deutscher Bundestag 2002: 38 f.). Dem bürgerschaftlichen Engagement kommt insbesondere unter veränderten demografischen Bedingungen eine große Rolle zu (u. a. Bieker et al. 2004; Voß et al. 2011; BMFSFJ 2016). In diesem Kontext werden Wanderungsbewegungen häufig als $\mathrm{Zu}$ - und Abwanderungen thematisiert und im Hinblick auf ihre Bedeutung für eine zukunftsfähige Gemeindeentwicklung evaluiert; multilokale Arrangements sind selten im Fokus (vgl. Stöber 2010). Die Herausforderungen der Bewältigung der demografischen Entwicklung in ländlichen Räumen lassen sich nicht ausschließlich durch einen weiteren Ausbau der technischen Infrastruktur en oder das Vertrauen in zukünftige Möglichkeiten der Digitalisierung bewältigen. In jüngerer Zeit kommt den Diskursen um eine Selbstresponsibilisierung (Steinführer 2014: 14) eine größere Bedeutung zu, die dann häufig mit Fragen der Sozialen Verpflichtung verknüpft werden. Hierbei ist primär zu berücksichtigen, dass die Realisierung einer sozialen Verantwortung in drei Dimensionen geschehen muss: Normative Verpflichtungen, zeitlicher Einsatz 
und die Nutzung finanzieller Ressourcen spielen hier gleichmäßig eine wichtige Rolle (Rossi 2001: 97). Untersuchungen haben bereits gezeigt, dass Multilokale multiple Ortsbindungen ausbilden und sich somit an mehreren Orten beheimatet fühlen (vgl. Duchêne-Lacroix 2015; Lange 2018; Nadler 2020). Das wiederum kann sich unterschiedlich auf die Engagementtätigkeiten der Multilokalen auswirken. So gibt es sowohl Multilokale, die ihr Engagement aufgrund zeitlicher Engpässe aufgeben als auch Einzelfälle, bei denen eine Tätigkeit auch am nachrangig erschlossenen Ort ausgeübt wird (Lange 2018). Insbesondere aufgrund der stark eingeschränkten Freizeit durch vielfach lange Fahrtwege zwischen ihren Orten, ist eher davon auszugehen, dass Multilokale im Hinblick auf bürgerschaftliches Engagement, wie beispielsweise auch die Gruppe der Tagespendler*innen, stark eingeschränkt sind (ebd.). Letztendlich bleibt festzuhalten, dass so individuell wie sich die mehrörtigen Lebensweisen darstellen, so komplex und vielfältig auch die verschiedenen Haltungen gegenüber freiwilligen Tätigkeiten außerhalb von Beruf und Familie sind.

Die hierbei vorhandenen Schnittstellen zum Phänomen multilokaler Anwesenheitsarrangements berühren aber nicht nur die zeitliche Dimension, sondern bedürfen auch einer Einbettung in Fragen der Gemeindeentwicklung (Mtika/ Kistler 2017: 90). In diesem Kontext stellen die nachfolgenden vier Entwicklungsfaktoren wichtige Aspekte dar, die die Zusammenhänge zwischen normativ-erwünschten und tatsächlich möglichen Handlungen deutlich machen:

- Das Erkennen, Reflektieren und die Umsetzung von Innovationen: Multilokale verfügen durch die Anwesenheiten an verschiedenen Orten vielfach über ein breites Wissen und Ideen, die sie andernorts erlernt haben und die sie am Ausgangsort einbringen und der Gemeinschaft zu Gute kommen lassen können (Wissen- und Ideentransfer).

- Die Entwicklung und Verfestigung von Werten: Multilokalität ermöglicht immer auch eine Form der Stabilität (bspw. als Möglichkeit einen vollständigen Umzug abzuwenden) und kann somit zur Beständigkeit einer Gemeinschaft beitragen (zumal im Falle eines multilokal lebenden Haushaltes es stets dauerhaft anwesende Personen am Ausgangsort gibt, die die Gemeinschaft und den Ort beleben können). Das Leben an mehreren Orten und insbesondere das stetige Zurückkehren an den Ausgangsort kann Heimatgefühl und Verbundenheit verfestigen.

- Der organische Aufbau einer Organisation im Hinblick auf eine Bewertung der vorhandenen Ressourcen: Multilokale als Netzwerker*innen an mehreren Orten können ihre Ressourcen an den Orten einbringen. Dabei sind physische Anwesenheiten oft nur phasenweise möglich. Gleichzeitig ist zu vermuten, dass 
Multilokale die beiden Anforderungen der Flexibilität und Reflexivität bei der Entwicklung der Organisation in besonderer Weise ausfüllen können, da beide Aspekte zu ihrem Lebensentwurf gehören. Digitale Kommunikation hingegen kann helfen, Ressourcen in digitalen „Anwesenheiten“ nutzbar zu machen.

- Die Integration aller Maßnahmen in ein Gesamtentwicklungskonzept: Multilokalität als gesamtgesellschaftliches Phänomen gilt es in der Entwicklung von Kommunen mitzudenken und zu berücksichtigen. In Abhängigkeit der jeweiligen kommunalen Rahmenbedingungen können insbesondere ländliche Räume eine besondere Betroffenheit aufweisen, die vielschichtige positive und negative Konsequenzen bedeuten können.

Unter dem Stichwort „Caring Communities“ oder "Sorgende Gemeinden“ werden Versuche einer synergetischen und koproduktiven Gestaltung der Aktivitäten von professionellen Dienstleistern, Familienangehörigen, sonstigen ehrenamtlich Engagierten und staatlicher bzw. nicht-staatlicher Institutionen subsumiert und als ein Lösungsansatz für die sorge- und pflegespezifischen Herausforderungen ländlicher Räume anerkannt (Klie 2016). Hierbei werden multilokale Arrangements für Forschung und Praxis relevant, als dass mit ihnen Einschränkungen in der Umsetzung dieses Konzepts auf der „Familienseite“ verbunden sind; zusätzlich ist darauf zu verweisen, dass Selbstresponsibilisierungsansätze in der Pflege ihrerseits wieder zu Multilokalität führen können, wenn Pflegekräfte an zwei Orten in die Pflege von Patient*innen und von eigenen Familienangehörigen eingebunden sind (vgl. Bowlby/McKie 2019; Fischer 2020).

\section{Multilokaler Lebensalltag in ländlichen Räumen: Fünf Beispiele aus dem Landkreis Diepholz}

Auch in der Fallstudie, dem Landkreis Diepholz, sind die Motive und Gründe für ein Leben an mehreren Orten sehr unterschiedlich und greifen häufig ineinander (vgl. Beitrag Othengrafen/Greinke/Lange/Seitz in diesem Band (Kap. 1)). Viele Formen und Dimensionen sind bislang noch nicht in das gesellschaftliche Bewusstsein gerückt (Hilti 2013: 11), sind aber über nahezu alle Gesellschaftsschichten verteilt (Dittrich-Wesbuer/Föbker 2013: 392). Zudem tritt das Phänomen in nahezu allen Lebensphasen auf: Sowohl Kinder als auch junge Erwachsene bis hin zu Erwachsenen und Personen im Ruhestand führen ein Leben an mehreren Orten (Hilti 2013: 22; Dittrich-Wesbuer/Kramer 2014: 46). Oft ist die fluide Lebensweise gekennzeichnet von Entwicklungs- und Veränderungsdynamiken (Dittrich-Wesbuer/Kramer 2014: 46).

Da aufgrund der Vielfalt und Komplexität des Phänomens noch nicht alle Formen der mehrörtigen Lebensweise erfasst sind (Schad/Duchêne-Lacroix 2013: 360) und dies ggf. auch gar nie möglich sein wird, werden nachfolgend 
beispielhafte (fiktive) Personenbeschreibungen dargestellt, um einen Einblick in die Ursachen und Motive multilokaler Lebensweisen in ländlichen Räumen am Beispiel des Landkreises Diepholz zu bieten.

Bernd ist 43 Jahre alt und lebt mit seiner Frau und zwei Kindern, die fünf und neun Jahre alt sind, in Köln. Nach seinem Abitur hat er Informatik in München und Berlin studiert. Seine Frau hat er während des Studiums kennengelernt. Beide sind gemeinsam nach Köln in eine Mietwohnung mit $120 \mathrm{~m}^{2}$ gezogen. Dort leben sie mit ihren Kindern recht innenstadtnah. Ihre Kinder besuchten in der Stadt den Kindergarten und nun auch die Schule. Bernd arbeitet seit zehn Jahren in einem international tätigen Unternehmen, dessen Hauptsitz in einer ländlich geprägten Kommune, der Samtgemeinde (SG) Barnstorf, liegt und welches mehrere Standorte im In- und Ausland hat. Bernd kann zeitweise von zu Hause oder unterwegs arbeiten. Zunächst war Bernd im Betrieb in Köln tätig. Später wurde er für eine kurze Projektarbeit in die SG Barnstorf delegiert. Geplant war, dass er nach der Projekttätigkeit wieder in Köln arbeitet. Da sich aber die Projektlaufzeit verlängert und gleichzeitig ein neues Projekt in der SG Barnstorf gestartet ist, lebt er seit ca. sechs Jahren multilokal. In der SG Barnstorf hat er sich eine kleine Mietwohnung angemietet, die nah am Bahnhof liegt und von der der Betrieb fußläufig erreicht werden kann. Dort ist er i. d. R. zwischen Dienstag bis Donnerstag; Montag und Freitag kann er im Homeoffice in Köln arbeiten. Bernd ist nun schon länger Projektleiter und wird aufgrund seines Know-hows öfter an verschiedene Betriebsstandorte delegiert, um dort Projekte zu leiten. Mit dem Leben an mehreren Orten hat er sich angefreundet. Bernd wäre aber lieber wieder ausschließlich in Köln tätig, um nicht nur an den Wochenenden bei seiner Familie und bei Freunden zu sein.

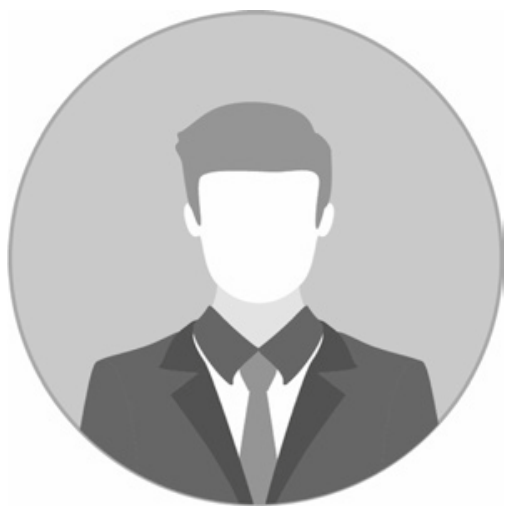

Bernd, 43 Jahre, Informatiker 
Achmed ist 19 Jahre alt und lebt seit seiner Geburt in einer ländlich geprägten Kommune, der SG Kirchdorf. Er ist dort aufgewachsen und zur Schule gegangen. Nach seinem Realschulabschluss hat er eine Ausbildung zum Bürokaufmann bei einem kleinen Unternehmen im Nachbarort begonnen. Derzeit lebt er noch bei seinen Eltern im Haus in einer kleinen Einliegerwohnung. Zum Betrieb fährt er montags bis donnerstags mit seinem kleinen PKW. Während der Ausbildung muss Achmed regelmäßig freitags zur Berufsschule, die im nächstgelegenen Oberzentrum, der Stadt Bremen, liegt und mit dem ÖPNV nur schwer zu erreichen ist. Dorthin fährt er in einer Fahrgemeinschaft mit anderen Auszubildenden aus den Nachbarorten. Für den Blockunterricht, der zweimal im Jahr für ca. ein bis zwei Monate in der Berufsschule stattfindet, wohnt Achmed in einem kleinen Zimmer in einem Wohnheim, welches von seinem Unternehmen angemietet wird. Seine Freunde sieht er in der Zeit des Blockunterrichts eher selten, weil er in der Woche nicht zu Hause ist und an den Wochenenden lernen muss.

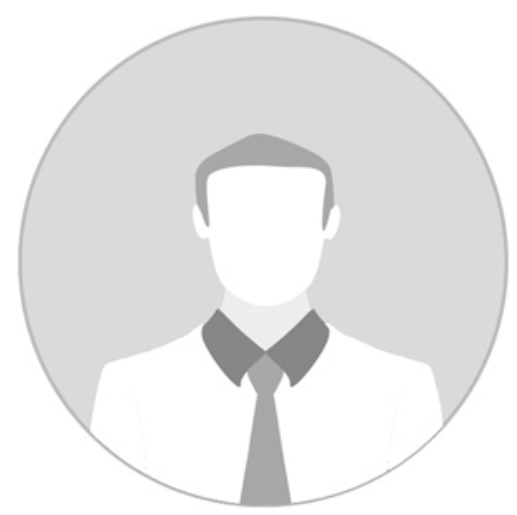

Achmed, 19 Jahre, Auszubildender

Luisa ist sechs Jahre alt und in einer ländlich geprägten Kommune, der SG Rehden, aufgewachsen. Dort besucht sie auch die erste Klasse. Ihre Eltern haben sich vor zwei Jahren getrennt. Nun lebt sie innerhalb der Woche bei ihrem Vater in ihrem ,alten“ Kinderzimmer. An den Wochenenden wohnt Luisa bei ihrer Mutter, die aus dem gemeinsamen Haus in eine kleinere Wohnung in der Nachbarschaft gezogen ist. In der Wohnung ihrer Mutter hat Luisa ihr eigenes 
Kinderzimmer. Weil die beiden Unterkünfte nicht weit entfernt voneinander liegen, kann sie zu Fuß oder mit dem Fahrrad zwischen ihnen pendeln. Deshalb ist Luisa auch oft innerhalb der Woche bei ihrer Mutter oder ihrem Vater und wechselt hin und her.

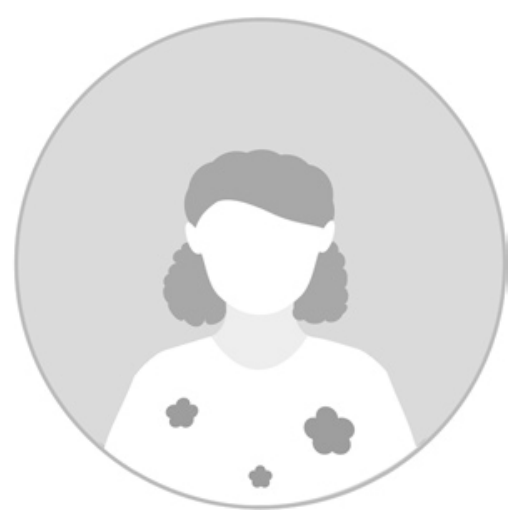

Luisa, 6 Jahre, Schülerin

Alexandra und Martin sind 65 Jahre alt und seit einem Jahr im Ruhestand. Beide leben seit 40 Jahren gemeinsam in Hamburg in einer $90 \mathrm{~m}^{2}$ Eigentumswohnung. Diese haben sie sich nach ihrer Hochzeit gekauft. Beide waren lange Jahre in Hamburg bei einem größeren Unternehmen in der Produktion tätig. Durch Zufall haben sie von ca. zehn Jahren Urlaub in der SG Altes Amt Lemförde am Dümmer See gemacht. Dort hat es ihnen so gut gefallen, dass sie sich einen Dauerstellplatz auf einem Campingplatz gemietet haben. Diesen besitzen sie nun seit acht Jahren. Seit sie vor zwei Jahren in den Ruhestand gegangen sind, verbringen sie den ganzen Sommer als Dauercamper*innen auf dem Campingplatz. An den Wochenenden bekommen sie dort gelegentlich Besuch von Freunden oder ihren Enkelkindern. Da es ihnen im Winter aber zu kalt ist, verbringen sie diese Jahreszeit in ihrer Wohnung in Hamburg. Die An- und Abreise gestalten sie ausschließlich mit dem Auto, weil sie oft viel Gepäck für ihre langen Aufenthalte mitnehmen. 


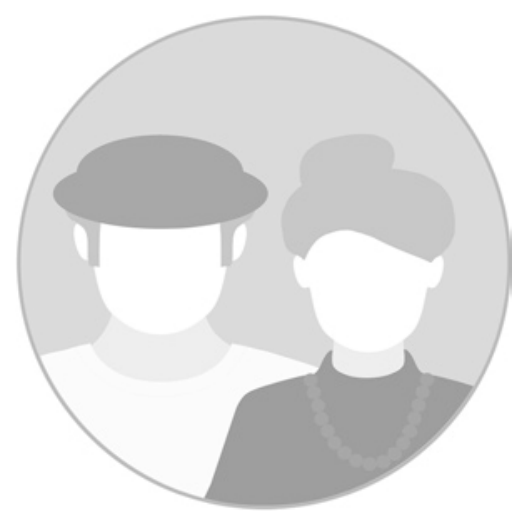

Alexandra und Martin, 65 Jahre, im Ruhestand

Claudio ist 28 Jahre alt und lebt in der Stadt Diepholz, einer ländlich geprägten Kommune. Er hat nach dem Abitur, welches er in Diepholz gemacht hat, ein Studium in Kiel begonnen. Seit fünf Jahren ist er Anlagenbauer in einem mittelständigen Unternehmen im Landkreis. Während des Studiums hat er Vanessa kennengelernt, mit der er seit acht Jahren zusammen ist. Sie führen eine Wochenendbeziehung: Die Wochenenden verbringen sie abwechselnd in Diepholz bei ihm oder in Delmenhorst bei ihr. In Diepholz ist Claudio in der Freiwilligen Feuerwehr engagiert und aufgrund seiner Beziehung alle zwei Wochen nicht vor Ort, um die Tagesalarmbereitschaft zu sichern. Er hat schon oft darüber nachgedacht, in Delmenhorst eine Doppelmitgliedschaft bei der Feuerwehr anzutreten. Aufgrund der geringen Zeitverfügbarkeit hat Claudio es bislang aber noch nicht realisiert. An den Wochenenden bei Vanessa möchten die beiden lieber gemeinsam ihre Freizeit verbringen, als zusätzlich noch ein Engagement auszuüben. 


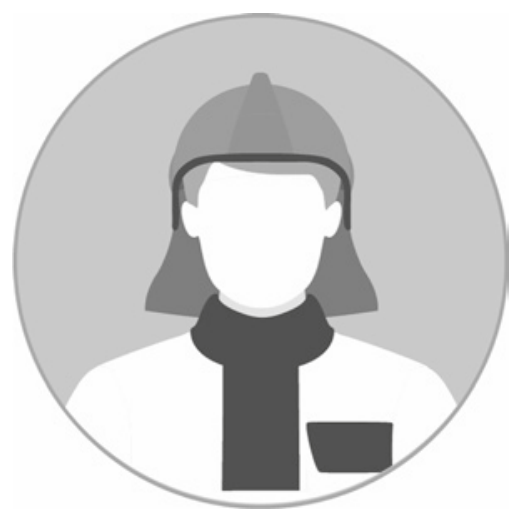

Claudio, 28 Jahre, Anlagenbauer

\subsection{Multilokalität als Phänomen in ländlichen Räumen}

Die im Beitrag aufgezeigte Diversität des Phänomens Multilokalität spiegelt sich u. a. in der Vielfalt an Motiven und Ausprägungen der Lebensformen wieder. Auch die spezifischen Anforderungen und Bedürfnisse der multilokal lebenden Personen an ihre Unterkünfte und Umgebungen unterstreichen diese Komplexität zusätzlich (ARL 2016: 9). Eine Interpretation von Multilokalität als zwischengeschaltete Phase im Lebensverlauf (Gehen oder Bleiben?) führt zur Frage der Einordnung von multilokalen Lebensstilen in Systematisierungsansätze der Zuwanderung in ländliche Räume. Der hier vorgenommene Rekurs auf Motivationen und Zwänge kann für Diskurse der Multilokalitätsforschung in ländlichen Räumen genutzt werden (Born 2007).

Multilokalität als Phänomen ist in ländlichen Räumen kein neues Phänomen. Die zuvor erläuterten Motive und Gründe für mehrörtige Lebensweisen gab es bereits vor vielen Jahrzehnten - auch in ländlichen Räumen. Mehrörtigkeit ist schon lange keine exklusive Lebensform mehr, sondern weiter verbreitet als oft angenommen wird. Aufgrund der gesamtgesellschaftlichen und globalen Entwicklungen ist zudem davon auszugehen, dass flexible und mobile Lebensformen auch zukünftig noch weiter zunehmen werden. Zumeist sind Informationen zu den verschiedenen Lebensarrangements jedoch nicht hinreichend bekannt, sodass Multilokalität als solche nicht oder nur unvollständig identifiziert wird. Das liegt 
unter anderem daran, dass dem Phänomen in der Vergangenheit wenig Beachtung geschenkt wurde und häufig auch heute noch Aussagen zum Vorkommen mehrörtig Lebender fehlen. Die sehr fluide Lebensführung von Multilokalen macht eine Erfassung und den Umgang damit nicht einfacher.

Über diese räumlichen Wirkungen von Multilokalität ist bislang jedoch besonders in ländlichen Räumen, wie dem südlichen Teil des Landkreises Diepholz, wenig bekannt. Um die Wirkungen adäquat analysieren zu können, ist es zunächst erforderlich, sich der Anzahl von Multilokalen in verschiedenen Beispielräumen anzunähern. So ist es möglich den quantitativen Effekt des Phänomens einzuordnen und aufbauend auf den Erkenntnissen allgemein übertragbare Ergebnisse und Strategien zum Umgang mit Multilokalität zu erarbeiten. Verschiedene erfolgreich erprobte methodische Ansätze zur Annäherung und zur Einordnung mehrörtiger Lebensweisen in ländlichen Räumen werden in den nachfolgenden Beiträgen von Albrecht/Dittrich-Wesbuer (Kap. 3) und Greinke/ Lange (Kap. 4) in diesem Band erläutert.

\section{Literatur}

Altrock, U./Güntner, S./Huning, S./Nuissl, H./Peters, D. (2005): Landliebe und Landleben. Planungsrundschau, Ausgabe 12: Landliebe - Landleben. Ländlicher Raum im Spiegel von Sozialwissenschaften und Planungstheorie, ARL 2016, 7-12.

ARL - Akademie für Raumforschung und Landesplanung (Hg.) (2016): Multilokale Lebensführung und räumliche Entwicklungen. Positionspapier aus der ARL 104. Hannover.

Baum, S./Weingarten, P. (2004): Typisierung ländlicher Räume in Mittel- und Osteuropa. In: Europa Regional. 12.2004 (3), 149-158.

Bundesinstitut für Bau-, Stadt und Raumforschung (BBSR) im Bundesamt für Bauwesen und Raumordnung (BBR) (Hg.) (2010): Laufende Raumbeobachtung Raumabgrenzung. Online verfügbar unter: https://www.bbsr.bund.de/BBSR/DE/ Raumbeobachtung/Raumabgrenzungen/deutschland/gemeinden/Raumtypen2010_vbg/ raumtypen2010_node.html.

Beck, U. (1986): Risikogesellschaft. Auf dem Weg in eine andere Moderne. Frankfurt a.M.: Suhrkamp.

Beck, U./Beck-Gernsheim, E. (1994): Individualisierung in modernen Gesellschaften: Perspektiven und Kontroversen einer subjektorientierten Soziologie. In: Beck, U./ Beck-Gernsheim, E. (Hg.): Riskante Freiheiten: Individualisierung in modernen Gesellschaften. Frankfurt a.M.: Suhrkamp, 10-39.

Becker, E./Gualini, E./Runkel, C./Strachwitz Graf, R. (Hg.) (2010): Stadtentwicklung, Zivilgesellschaft und bürgerschaftliches Engagement. Stuttgart: Lucius \& Lucius, Maecenata-Schriften, 6.

Bieker, S./Knieling, J./Othengrafen, F./Sinning, H. (2004): Kooperative Stadt-Region 2030. Braunschweig. Beiträge zu STADT+UM+LAND 2030 Region Braunschweig, Bd. 13. 
Blotevogel, H. H. (2005): Metropolräume und ländliche Räume - eine Solidargemeinschaft? Universität Dortmund, Fakultät Raumplanung. Schriftliche Fassung eines Vortrags im Rahmen des Fachkongresses „Leitlinien der niedersächsischen Landesentwicklungspolitik“ am 24.11.2005. Hannover.

Bundesministerium für Ernährung und Landwirtschaft (Hg.) (2019): Landatlas. Online verfügbar unter: https://www.bmel.de/DE/Laendliche-Raeume/InformationsportalZukunftLand/ Landatlas/landatlas_node.html.

BMFSFJ (2016): Zweiter Engagementbericht 2016. Demografischer Wandel und Bürgerschaftliches Engagement: Der Beitrag des Engagements zur lokalen Entwicklung. Berlin.

Bundesministerium für Verkehr, Bau und Stadtentwicklung (BMVBS)/Bundesinstitut für Bau-, Stadt und Raumforschung (BBSR) im Bundesamt für Bauwesen und Raumordnung (BBR) (Hg.) (2009): Ländliche Räume im demografischen Wandel. BBSROnline-Publikation 34/2009. Bonn.

Bundesministerium für Verkehr und digitale Infrastruktur (Hg.) (2015): Aktionsprogramm regionale Daseinsvorsorge. Projektassistenz Umsetzungsphase. BMVI-OnlinePublikation 04/2015, Bearbeiter: Dehne, P./Kaether, J./Neubauer, A.

Born, K. M. (2011): Ländliche Räume in Deutschland. Differenzierungen, Entwicklungspfade und -brüche. Geographische Rundschau 63 (3), 4-11.

Born. K. M. (2007): Zuwanderungen in periphere ländliche Räume. Eine Chance? In: Schmied, D./Henkel. G. (Hg.): Leerstände von Gebäuden in Dörfern - Beginn der Dorfauflösung oder Chancen durch Umsetzung? Göttingen,19-36.

Bowlby, S./McKie, L. (2019): Care and Caring: An ecological framework. Area 51 (3), 532-539.

Breyer, F. (1970): Die Wochenendpendler des Bayrischen und Östlichen Oberpfälzer Waldes. WGI-Berichte zur Regionalforschung, Bd. 4.

Danielzyk, R./Dittrich-Wesbuer, A. (2020): Multilokalität in der Stadt- und Regionalentwicklung. In: Danielzyk, R./Dittrich-Wesbuer, A./Hilti, N./Tippel, C. (Hg.) (2020): Multilokale Lebensführungen und räumliche Entwicklungen - ein Kompendium. Hannover. Forschungsberichte der ARL 13. URN : https://nbn-resolving.de/ urn:nbn:de:0156-09764, 196-202.

Deutscher Bundestag (Hg.) (2002): Bericht der Enquete-Kommission „Zukunft des Bürgerschaftlichen Engagements“, Bürgerschaftliches Engagement: Auf dem Weg in eine zukunftsfähige Bürgergesellschaft. Drucksache 14/8900, 03.06.2002.

Dienel, H.-L. (2009): Multilokales Wohnen zwischen Kontrasträumen. Befunde und Konzepte zu individuellen und politischen Raumpartnerschaften. In: Informationen zur Raumentwicklung, Heft 1/2, 117-123.

Dirksmeier, P. (2012): Multilokalität als Abwesenheit: Eine Herausforderung für landschaftliche attraktive ländliche Räume - das Beispiel Tegernsee/Stadt. Europa Regional, 10/2010 2-3, 60-70.

Dirksmeier, P. (2019): Kulturgeografie. In: Nell, W./Weiland, M. (Hg.): Dorf. Ein interdisziplinäres Handbuch. Berlin, 27-31.

Dittrich-Wesbuer, A./Eichhorn, S./Tippel, C. (2014): Relevanz multilokaler Phänomene in der Stadtentwicklung. Ergebnisse aus Expertenbefragungen in Nordrhein-Westfalen. In: Hamman, P./Blanc, M./Duchêne-Lacroix, C./ Freytag, T./Kramer, C. (Hg.) 
(2014): Questionner les mobilités résidentielles à l'aune de la multilocalité. Strasbourg: Néothèque Éditions, 275-357.

Dittrich-Wesbuer, A./Föbker, S./Sturm, G. (2015): Multilokales Wohnen: Empirische Befunde zur Verbreitung in Deutschland. In: Weichhart, P./Rumpolt, P. A. (Hg.) (2015): Mobil und doppelt sesshaft. Studien zur residenziellen Multilokalität. Wien: Abhandlungen zur Geographie und Regionalforschung Bd. 18, 121-143.

Dittrich-Wesbuer, A./Föbker, S. (2013): Multilokales Wohnen - Verbreitung und Formen in Deutschland. In: Scheiner, J./Blotevogel, H. H./Frank, S./Holz-Rau, C./ Schuster, N. (Hg.) (2013): Mobilitäten und Immobilitäten: Menschen - Ideen - Dinge - Kulturen - Kapital. Konferenzband: Dortmunder Konferenz Raum- und Planungsforschung: Mobilitäten und Immobilitäten 1/2012, Blaue Reihe, Bd. 142, 391-402.

Dittrich-Wesbuer, A./Kramer, C. (2014): Heute hier - morgen dort. Residenzielle Multilokalität in Deutschland. In: Geographische Rundschau 11I 2014, 46-52.

Dittrich-Wesbuer, A./Plöger, J. (2013): Multilokalität und Transnationalität - Neue Herausforderungen für Stadtentwicklung und Stadtpolitik. Raumforschung und Raumordnung, Vol. 71, Issue 3, 195-205.

Dittrich-Wesbuer/Hilti 2020.

Duchêne-Lacroix, C. (2015): Archipel oder die Territorialität in der Multilokalität der Lebenswelt. In: Weichhart, P./Rumpolt, P. A. (Hg.) (2015): Mobil und doppelt sesshaft. Studien zur residenziellen Multilokalität. Wien: Abhandlungen zur Geographie und Regionalforschung Bd. 18, 218-239.

Duchêne-Lacroix, C. (2020): Typenbildung multilokalen Wohnens. In: Danielzyk, R./ Dittrich-Wesbuer, A./Hilti, N./Tippel, C. (Hg.) (2020): Multilokale Lebensführungen und räumliche Entwicklungen - ein Kompendium. Hannover. Forschungsberichte der ARL 13. URN : https://nbn-resolving.de/urn:nbn:de:0156-09764, 76-82.

Fachinger, U./Künemund, H. (Hg.) (2015): Gerontologie und ländlicher Raum. Lebensbedingungen, Veränderungsprozesse und Gestaltungsmöglichkeiten. Vechtaer Beiträge zur Gerontologie. Wiesbaden: Springer VS Verlag.

Fischer, T. (2020): Familien: Multilokale Lebensführung bei Ausbildung und Pflege. In: Danielzyk, R./Dittrich-Wesbuer, A./Hilti, N./Tippel, C. (Hg.) (2020): Multilokale Lebensführungen und räumliche Entwicklungen - ein Kompendium. Hannover. Forschungsberichte der ARL 13. URN : https://nbn-resolving.de/urn:nbn:de:0156-09764, 119-126.

Franzen, N./Hahne, U./Hartz, A./Kühne, O./Schafranski, F./ Spellerberg, A./Zeck, H. (2008): Herausforderung Vielfalt - Ländliche Räume im Struktur- und Politikwandel. E-Paper der ARL Nr. 4. Hannover.

Greinke, L./Lange, L./Othengrafen, F. (2018): Multilokales Wohnen in ländlichen Räumen - Chancen und Herausforderungen. PlanerIn 18 (3), 44-46.

Greinke, L./ Hilti, N. (2019): Temporär genutzte Räume von berufsbedingt multilokal Lebenden. Herausforderungen und Potenziale für betriebliche und regionale Entwicklungen in ländlichen Räumen Niedersachsens. Raumforschung und Raumordnung 78 (1): $1-17$.

Hahne, U. (2009): Zukunftskonzepte für schrumpfende ländliche Räume. Von dezentralen und eigenständigen Lösungen zur Aufrechterhaltung der Lebensqualität und zur Stabilisierung der Erwerbsgesellschaft. Neues Archiv für Niedersachsen. Zeitschrift für Stadt-, Regional- und Landesentwicklung. Heft 1/2009, 2-25. 
Henkel, G. (2009): Stärken und Schwächen unserer Dörfer - Wie könnte ein Fitnessprogramm für die Zukunft aussehen In: Heimatpflege in Westfalen - 22. Jg., 4/2009, $1-10$.

Henkel, G. (2010): Stärken und Schwächen unserer Dörfer - Wie könnte ein Fitnessprogramm für die Zukunft aussehen? In: Flächenmanagement und Bodenordnung, Heft 2, 54-61.

Henkel, G. (2016): Geschichte und Gegenwart des Dorfes. In: BPB - Bundeszentrale für politische Bildung (Hg.) (2016): Land und Ländlichkeit. Aus Politik und Zeitgeschichte. Zeitschrift der Bundeszentrale für politische Bildung. 66. Jg., 46-47/2016, $10-16$.

Herrmann, C. (2005): Selbstorganisierte Entgrenzung der Arbeitszeit? Flexible Arbeitszeiten und neue Formen der Arbeitsorganisation. In: Seifert, H. (Hg.): Flexible Zeiten in der Arbeitswelt. Frankfurt am Main, 216-244.

Hesse, M./Scheiner, J. (2007): Räumliche Mobilität im Kontext des sozialen Wandels: eine Typologie multilokalen Wohnens. Geographische Zeitschrift 95, 3, 138-154.

Hilti, N. (2009): Multilokales Wohnen: Bewegungen und Verortungen. Informationen zur Raumentwicklung, Heft 1/2, 77-86.

Hilti, N. (2013): Lebenswelten multilokal Wohnender. Eine Betrachtung des Spannungsfeldes von Bewegung und Verankerung. Wiesbaden: Springer Fachmedien.

Hilti, N. (2020): (Multilokales) Wohnen. In: Danielzyk, R./Dittrich-Wesbuer, A./Hilti, N./ Tippel, C. (Hg.) (2020): Multilokale Lebensführungen und räumliche Entwicklungen - ein Kompendium. Hannover. Forschungsberichte der ARL 13. URN : https://nbnresolving.de/urn:nbn:de:0156-09764, 222-228.

Hilti, N./Huber, A. (2020): Dauercamping als multilokale Wohnform. In: Danielzyk, R./ Dittrich-Wesbuer, A./Hilti, N./ippel, C. (Hg.) (2020): Multilokale Lebensführungen und räumliche Entwicklungen - ein Kompendium. Hannover. Forschungsberichte der ARL 13. URN : https://nbn-resolving.de/urn:nbn:de:0156-09764, 254-259.

Huchler, N. (2020): Airlines und die Multilokalität des Flugpersonals. In: Danielzyk, R./ Dittrich-Wesbuer, A./Hilti, N./Tippel, C. (Hg.) (2020): Multilokale Lebensführungen und räumliche Entwicklungen - ein Kompendium. Hannover. Forschungsberichte der ARL 13. URN : https://nbn-resolving.de/urn:nbn:de:0156-09764, 315-321.

Jacoby, C./Wappelhorst, S. (Hrsg.) (2016): Potenziale neuer Mobilitätsformen und technologien für eine nachhaltige Raumentwicklung. Arbeitsberichte der ARL 18. Hannover.

Kahle, I./Schäfer, D. (2005): Ehrenamt und bürgerschaftliches Engagement. Ergebnisse der Zeitbudgeterhebung 2001/2002. Statistisches Bundesamt (Hg.): Wirtschaft und Statistik, Heft 4/2005, Wiesbaden, 311-317.

KIT - Karlsruher Institut für Technologie (2015): Residentielle Multilokalität und ihre Folgen. URN: https://www.kit.edu/kit/16379.php [14.10.2015].

Klie, T. (2016): Caring Community. Auf dem Weg in eine sorgende Gemeinschaft? In: Zimmermann, H.-P./Kruse, A./Rentsch, T. (Hg.): Kulturen des Alterns. Plädoyers für ein gutes Leben bis ins hohe Altern. Frankfurt, 269-286.

Kramer, C. (2020): Multilokales Leben von Studierenden in Karlsruhe. In: Danielzyk, R./ Dittrich-Wesbuer, A./Hilti, N./Tippel, C. (Hg.) (2020): Multilokale Lebensführungen und räumliche Entwicklungen - ein Kompendium. Hannover. Forschungsberichte der ARL 13. URN : https://nbn-resolving.de/urn:nbn:de:0156-09764, 278-285. 
Küpper, P. (2010): Regionale Reaktionen auf den demographischen Wandel in dünn besiedelten, peripheren Räumen: Ergebnisse einer deutschlandweiten Befragung. Raumforschung und Raumordnung, Vol. 68, 169-180.

Lange, L. (2018): Leben an mehreren Orten. Multilokalität und bürgerschaftliches Engagement in ländlich geprägten Räumen Niedersachsens. Berlin: LIT Verlag.

Leber, N./Kunzmann, K. R. (2006) Entwicklungsperspektiven ländlicher Räume in Zeiten des Metropolenfiebers. disP - The Planning Review, Vol. 42, Issue 166: Prospects for Rural Regions, 58-70.

Leubert, N. (2015): Heute hier. Morgen dort. Chancen und Herausforderungen multilokaler Lebensstile in Wolfsburg. Graue Reihe des Instituts für Stadt- und Regionalplanung an der TU Berlin, 47, Berlin: Universitätsverlag der TU Berlin.

Leubert, N. (2020): Annäherung an die berufsbedingt Multilokalen in Wolfsburg. In: Danielzyk, R./Dittrich-Wesbuer, A./Hilti, N./Tippel, C. (Hg.) (2020): Multilokale Lebensführungen und räumliche Entwicklungen - ein Kompendium. Hannover. Forschungsberichte der ARL 13. URN : https://nbn-resolving.de/urn:nbn:de:0156-09764, 243-252.

Litter, V./Stawarz, N. (2013): Alltagsarrangements und räumliche Mobilität - am Fallbeispiel des universitären Mittelbaus der Technischen Universität Chemnitz. In: Scheiner, J./Blotevogel, H. H./Frank, S./Holz-Rau, C./Schuster, N. (Hg.) (2013): Mobilitäten und Immobilitäten: Menschen - Ideen - Dinge - Kulturen - Kapital. Konferenzband: Dortmunder Konferenz Raum- und Planungsforschung: Mobilitäten und Immobilitäten 1/2012, Blaue Reihe, Bd. 142, 403-416.

Menzl, M. (2020): Wohnen zwischen Multilokalität und Ortsbindung - das Beispiel der HafenCity Hamburg. In: Danielzyk, R./Dittrich-Wesbuer, A./Hilti, N./Tippel, C. (Hg.) (2020): Multilokale Lebensführungen und räumliche Entwicklungen - ein Kompendium. Hannover. Forschungsberichte der ARL 13. URN : https://nbn-resolving. de/urn:nbn:de:0156-09764, 235-242.

Milbert, A. (2016): Landflucht? Gesellschaft in Bewegung. Informationen zur Raumentwicklung, Heft 2/2016, 105-107.

Mtika, M. M./Kistler, M. (2017): Contiguous community development. Journal of Rural Studies 51, 83-92.

Nadler, R. (2014): Plug\&Play Places. Lifeworlds of Multilocal Creative Knowledge Workers. Warsaw/Berlin: De Gruyter Open Ltd.

Nadler, R. (2020): Plug\&Play Places: Über die Bespielbarkeit von Orten in multilokalen Lebenswelten. In: Danielzyk, R./Dittrich-Wesbuer, A./Hilti, N./Tippel, C. (Hg.) (2020): Multilokale Lebensführungen und räumliche Entwicklungen - ein Kompendium. Hannover. Forschungsberichte der ARL 13. URN : https://nbn-resolving.de/ urn:nbn:de:0156-09764, 308-314.

Nadler, R./Montanari, G. (2013): Der Wohnbegriff in der Multilokalitätsforschung Überlegungen anhand der Beispielgruppen Großeltern in Nachtrennungsfamilien und kreative WissensarbeiterInnen. In: Scheiner, J./Blotevogel, H. H./Frank, S./HolzRau, C./Schuster, N. (Hg.) (2013): Mobilitäten und Immobilitäten: Menschen - Ideen - Dinge - Kulturen - Kapital. Konferenzband: Dortmunder Konferenz Raum- und Planungsforschung: Mobilitäten und Immobilitäten 1/2012, Blaue Reihe, Bd. 142, 417430.

Neu, C. (Hg.) (2009): Daseinsvorsorge. Eine gesellschaftswissenschaftliche Annäherung. Wiesbaden. 
Oel, H.-U. (2009): Das Ende der Ressorthoheit? Der demografische Wandel erfordert neue Politikkonzepte und neue Entscheidungsstrukturen. Bundesministerium für Verkehr, Bau und Stadtentwicklung (BMVBS)/Bundesinstitut für Bau-, Stadt und Raumforschung (BBSR) im Bundesamt für Bauwesen und Raumordnung (BBR) (Hrsg.) (2009): Ländliche Räume im demografischen Wandel. BBSR-Online-Publikation 34/2009, 124-131.

Pahl-Weber, E./Roskamm, N. (2007): Weniger Menschen - andere Stadt? Zum Umgang mit städtischen Schrumpfungsprozessen im Westen Deutschlands. In: Giseke, U./ Spiegel, E. (Hg.) (2007): Stadtlichtungen. Irritationen, Perspektiven, Strategien. Basel: Birkhäuser Verlag AG und Gütersloh: Bauverlag BV GmbH, 85-108.

Petzold, K. (2009): Multilokale Identifikation. Ein theoretisches Modell zur Entstehung kognitiv-emotionaler Bindungen an mehrere Orte. In: Wolfgang, A./ Obkircher, S./Saurwein, K. (Hg) (2009): „DOKONARA 2008. 2. internationales DoktorandInnenkolleg Nachhaltige Raumentwicklung". 157-172.

Petzold, K. (2011): Die europäische Stadt und multilokale Lebensformen: Eine Beziehung mit Zukunft? In: Frey, O./Koch, F. (Hg.) (2011): Die Zukunft der europäischen Stadt. Stadtpolitik, Stadt-planung und Stadtgesellschaft im Wandel. 153-172.

Petzold, K. (2013): Multilokalität als Handlungssituation. Lokale Identifikation, Kosmopolitismus und ortsbezogenes Handeln unter Mobilitätsbedingungen. Buchreihe: Forschung und Entwicklung in der Analytischen Soziologie. Wiesbaden: Springer Fachmedien.

Petzold, K. (2020): Multilokalität, raumbezogene Einstellungen und lokales Handeln. In: Danielzyk, R./Dittrich-Wesbuer, A./Hilti, N./Tippel, C. (Hg.) (2020): Multilokale Lebensführungen und räumliche Entwicklungen - ein Kompendium. Hannover. Forschungsberichte der ARL 13. URN : https://nbn-resolving.de/urn:nbn:de:0156-09764, 286-291.

Peuckert, R. (2012): Familienformen im sozialen Wandel. Wiesbaden: Springer Fachmedien.

Plieninger, T./Bens, O./Hüttl, R. (2006): Landwirtschaft und Entwicklung ländlicher Räume. In: Bundeszentrale für politische Bildung (BPB) (Hg.) (2016): Ländlicher Raum. Aus Politik und Zeitgeschichte. Zeitschrift der Bundeszentrale für politische Bildung. 66. Jg., 37/2016, 23-30.

Reuschke, D. (2009): Raum-zeitliche Muster und Bedingungen beruflich motivierter multilokaler Haushaltsstrukturen. In: Informationen zur Raumentwicklung, Heft 1/2, 31-42.

Reuschke, D. (2010a): Berufsbedingtes Pendeln zwischen zwei Wohnsitzen - Merkmale einer multilokalen Lebensform in der Spätmoderne. Comparative Population Studies Zeitschrift für Bevölkerungswissenschaft Jg. 35, 1., 135-164.

Reuschke, D. (2010b): Multilokales Wohnen. Raum-zeitliche Muster multilokaler Wohnarrangements von Shuttles und Personen in Fernbeziehung. Wiesbaden: Springer Fachmedien.

Rolshoven, J. (2007): Multilokalität als Lebensweise in der Spätmoderne. Schweizerisches Archiv für Volkskunde, Jg. 103, 157-179.

Rossi, A. S.(2001): Domains and Dimensions of Social Responsibility. A sociodemographic profile. In: Rossi, A. S. (Hg.): Caring and Doing for others. Social responsibility in the domains of family, work, and community. Chicago University Press. 97-125. 
Saxinger, G. (2020): Multilokalität als Normalisierungspraxis und synchrone Raumintegrationsleistung bei Fernpendelnden in der Erdöl- und Erdgasindustrie in Russlands Arktis. In: Danielzyk, R./Dittrich-Wesbuer, A./Hilti, N./Tippel, C. (Hg.) (2020): Multilokale Lebensführungen und räumliche Entwicklungen - ein Kompendium. Hannover. Forschungsberichte der ARL 13. URN : https://nbn-resolving.de/ urn:nbn:de:0156-09764, 322-328.

Schad, H./Duchêne-Lacroix, C. (2013): Multilokales Wohnen als hybride Praxis Implikationen der ,,mobilities studies“ und der Akteur-Netzwerk-Theorie. In: Scheiner, J./Blotevogel, H. H./Frank, S./Holz-Rau, C./Schuster, N. (Hg.) (2013): Mobilitäten und Immobilitäten: Menschen - Ideen - Dinge - Kulturen - Kapital. Konferenzband: Dortmunder Konferenz Raum- und Planungsforschung: Mobilitäten und Immobilitäten 1/2012, Blaue Reihe, Bd. 142, 359-374.

Scheiner, J. (2020): Verkehr: Bedeutung von Verkehrsangeboten für Multilokalität. In: Danielzyk, R./Dittrich-Wesbuer, A./Hilti, N./Tippel, C. (Hg.) (2020): Multilokale Lebensführungen und räumliche Entwicklungen - ein Kompendium. Hannover. Forschungsberichte der ARL 13. URN : https://nbn-resolving.de/ urn:nbn:de:0156-09764, 154-159.

Schier, M. (2009): Räumliche Entgrenzung von Arbeit und Familie. Die Herstellung von Familie unter Bedingungen von Multilokalität. Informationen zur Raumentwicklung, Heft 1/2, 55-66.

Schier, M. (2014): Multilokalität von Familie in Deutschland. Geographische Rundschau, 11, Themenheft „Multi- und Translokalität“, 10-17.

Sheller, M./Urry, J. (2006): The New Mobilities Paradigm. Environment and Planning, Vol. $38,207-226$.

Spiegel, E. (2007): Weniger - älter - bunter. Demographische Rahmenbedingungen der Stadtentwicklung. In: Giseke, U./Spiegel, E. (Hg.) (2007): Stadtlichtungen. Irritationen, Perspektiven, Strategien. Basel: Birkhäuser Verlag AG und Gütersloh: Bauverlag BV $\mathrm{GmbH}, 18-31$.

Steinführer, A. (2014): Wie lässt sich Schrumpfung gestalten. LandInForm 3, 14-15.

Stöber, S. (2010): Zwischen Landflucht und Leben(t)raum. Wege zu lebendigen Dörfern in Brandenburg. Weikersheim.

Sturm, G./Meyer, K. (2009): Was können die Melderegister deutscher Großstädte zur Analyse residenzieller Multilokalität beitragen? Informationen zur Raumentwicklung Heft $1 / 2,15-30$.

Sturm, G./Weiske, C. (2009): Multilokales Wohnen. Einführung. Informationen zur Raumentwicklung, Heft 1/2009: I-II.

Voß, W./Güldenberg, E./Jürgens, A./Kirsch-Stracke, R./Streibel, N. (2011) Modellprojekt Umnutzung landwirtschaftlicher Altgebäude und Hofanlagen als Beitrag zur Vitalisierung der Ortskerne. Abschlussbericht der wissenschaftlichen Begleitforschung der AG Dorfentwicklung an der Leibniz Universität Hannover.

Weber, G. (2010): Der ländliche Raum - Mythen und Fakten. Ländlicher Raum. OnlineFachzeitschrift des Bundesministeriums für Land- und Forstwirtschaft, Umwelt und Wasserwirtschaft. Jg. 2010, 1-11.

Weichhart, P (2020): Das Phänomen der residentiellen Multilokalität - Lifescapes, soziale Figurationen und raumstrukturelle Konsequenzen. In: Danielzyk, R./Dittrich-Wesbuer, A./Hilti, N./Tippel, C. (Hg.) (2020): Multilokale Lebensführungen und räumliche Ent- 
wicklungen - ein Kompendium. Hannover. Forschungsberichte der ARL 13. URN : https://nbn-resolving.de/urn:nbn:de:0156-09764, 42-49.

Weichhart, P. (2009): Multilokalität - Konzepte, Theoriebezüge und Forschungsfragen. Informationen zur Raumentwicklung, Heft 1/2, 1-14.

Weichhart, P./Rumpolt, P. A. (Hg.) (2015): Mobil und doppelt sesshaft. Studien zur residenziellen Multilokalität. Wien: Abhandlungen zur Geographie und Regionalforschung Bd. 18.

Weiske, C. (2013): Multilokale Arrangements als alltagspraktische Verknüpfungen von mobilen und immobilen Gütern. In: Scheiner, J./Blotevogel, H. H./Frank, S./HolzRau, C./Schuster, N. (Hg.) (2013): Mobilitäten und Immobilitäten: Menschen - Ideen - Dinge - Kulturen - Kapital. Konferenzband: Dortmunder Konferenz Raum- und Planungsforschung: Mobilitäten und Immobilitäten 1/2012, Blaue Reihe, Bd. 142, 343357.

Weiske, C./Petzold, K./Zierold, D. (2009): Multilokale Haushaltstypen. Bericht aus dem DFG-Projekt „Neue multilokale Haushaltstypen“ (2006-2008). Informationen zur Raumentwicklung, Heft 1/2009, 67-75.

Open Access Dieses Kapitel wird unter der Creative Commons Namensnennung 4.0 International Lizenz (http://creativecommons.org/licenses/by/4.0/deed.de) veröffentlicht, welche die Nutzung, Vervielfältigung, Bearbeitung, Verbreitung und Wiedergabe in jeglichem Medium und Format erlaubt, sofern Sie den/die ursprünglichen Autor(en) und die Quelle ordnungsgemäß nennen, einen Link zur Creative Commons Lizenz beifügen und angeben, ob Änderungen vorgenommen wurden.

Die in diesem Kapitel enthaltenen Bilder und sonstiges Drittmaterial unterliegen ebenfalls der genannten Creative Commons Lizenz, sofern sich aus der Abbildungslegende nichts anderes ergibt. Sofern das betreffende Material nicht unter der genannten Creative Commons Lizenz steht und die betreffende Handlung nicht nach gesetzlichen Vorschriften erlaubt ist, ist für die oben aufgeführten Weiterverwendungen des Materials die Einwilligung des jeweiligen Rechteinhabers einzuholen.

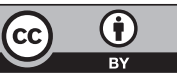

